\title{
Optically and electrically tunable Dirac points and Zitterbewegung in graphene-based photonic superlattices
}

\author{
Hanying Deng, ${ }^{1,2}$ Fangwei Ye,,${ }^{1,2, *}$ Boris A. Malomed, ${ }^{3}$ Xianfeng Chen,,${ }^{1,2}$ and Nicolae C. Panoiu ${ }^{4}$ \\ ${ }^{1}$ Department of Physics and Astronomy, Shanghai Jiao Tong University, Shanghai 200240, China \\ ${ }^{2}$ Key Laboratory for Laser Plasma (Ministry of Education), IFSA Collaborative Innovation Center, \\ Shanghai Jiao Tong University, Shanghai 200204, China \\ ${ }^{3}$ Department of Physical Electronics, School of Electrical Engineering, Faculty of Engineering, \\ Tel Aviv University, Tel Aviv 69978, Israel \\ ${ }^{4}$ Department of Electronic and Electrical Engineering, University College London, Torrington Place, London WC1E 7JE, United Kingdom
}

(Received 22 September 2014; revised manuscript received 19 April 2015; published 5 May 2015)

\begin{abstract}
We demonstrate that graphene-based photonic superlattices provide a versatile platform for electrical and all-optical control of photonic beams with deep-subwavelength accuracy. Specifically, by inserting graphene sheets into periodic metallodielectric structures, one can design optical superlattices that possess photonic Dirac points (DPs) at frequencies at which the spatial average of the permittivity of the superlattice $\bar{\varepsilon}$ vanishes. Similar to the well-known zero- $\bar{n}$ band gaps, we show that these zero- $\bar{\varepsilon}$ DPs are highly robust against structural disorder. We also show that, by tuning the graphene permittivity via the optical Kerr effect or electrical doping, one can induce a spectral variation of the DP exceeding $30 \mathrm{~nm}$, at mid-IR and THz frequencies. The implications of this wide tunability for the photonic Zitterbewegung effect in a vicinity of the DP are also explored.
\end{abstract}

DOI: 10.1103/PhysRevB.91.201402

PACS number(s): 73.20.Mf, 42.82.Et, 78.67.Pt, 78.68.+m

Introduction. One of the most daunting challenges one has to overcome in order to develop ultrafast active photonic nanodevices is the extremely narrow tunability of physical parameters of optical media. For example, while the conductivity of semiconductors can be varied by orders of magnitude with the help of impurity doping or thermally, the index of refraction of optical media, which is the main parameter defining their optical properties, can hardly be tuned by more than $0.5 \%$. Several approaches that address this key problem have been proposed, including the resort to plasmonic materials whose optical nonlinearity is enhanced by strong optical fields associated with the resonant excitation of surface-plasmon polaritons (SPPs) [1-3], the use of strongly anisotropic materials whose optical response can be controlled via the polarization of the excitation field $[4,5]$, reconfigurable metamaterials [6], embedding liquid crystals into a photoniccrystal matrix [7], and tuning the material refractive index via carrier injection [8]. While promising if targeted at specific applications, the efficiency of these methods is generally limited by high-power requirements, slow optical response, and reduced spectral tunability.

A recently introduced photonic platform that contains all the ingredients needed to achieve ultrafast broad optical tunability at the nanoscale is graphene [9-11]. Compared to the SPPs in metals, graphene plasmons are localized within much smaller regions, provide a much larger optical near-field enhancement, and can pass significantly longer distances [12,13]. Equally important, the conductivity of graphene and, implicitly, its electrical permittivity can be varied substantially, locally and ultrafast, by means of chemical doping or gate voltages [14-17]. For example, changing the Fermi level by a mere $1 \mathrm{eV}$ causes the relative variation of the real part of the permittivity, at the $10 \mu \mathrm{m}$ wavelength,

*fangweiye@sjtu.edu.cn by $250 \%$. Depositing graphene onto the surface of specific materials, which may be both dielectrics and metals, a variety of graphene-based layered nanostructures have been designed, and their implementations in functional devices have been demonstrated [14]. The most salient feature of these photonic superlattices is that, by incorporating graphene into their periodic structure, the band structure and thus their optical response can be dynamically tuned. This key property, in conjunction with relatively low loss and extremely tight confinement of the graphene plasmons, opens up promising applications of tunable graphene photonic superlattices (GPSs) at mid-IR and at $\mathrm{THz}$ wavelengths. In addition to these important practical considerations, GPSs can also play a major role at the fundamental level. Specifically, it has been suggested that electron-wave dynamics in graphene heterojunctions are in many ways similar to the propagation of optical beams in photonic superlattices containing positiveand negative-index materials [18], intriguing phenomena such as the Klein tunneling [19,20] and Zitterbewegung (ZB) effect [21-26] being observed in both physical systems.

In this Rapid Communication we demonstrate that, by including graphene sheets into the unit cell of metallodielectric superlattices, one can readily design GPSs that possess electrically and optically tunable Dirac points (DPs) and feature the ZB of optical beams propagating near the DPs. A remarkable property of these DPs is that they form when the spatial average of the permittivity of the superlattice vanishes, $\bar{\varepsilon}=0$, an immediate consequence of this fact being their remarkable robustness against structural disorder. To add specificity to these ideas, we consider, as an example of a GPS, the one-dimensional (1D) periodic structure depicted in Fig. 1(a). It has a three-layer unit cell, graphene-metaldielectric, the metallic and dielectric layers chosen in this work being silver and silicon, respectively. The dispersion relation of the structure is easily found by using the transfer-matrix method (see the Supplemental Material [27] for the derivation 
of this relation), and is given by

$$
\begin{aligned}
& \cos \left(k_{x} \Lambda\right) \\
& =\sigma\left(1-\frac{\varrho_{a b}^{2} \varrho_{b a}^{2}}{2 \varrho_{a b} \varrho_{b a}} \tan \kappa_{a} \tan \kappa_{b}-\frac{\varrho_{b c}^{2}+\varrho_{c b}^{2}}{2 \varrho_{b c} \varrho_{c b}} \tan \kappa_{b} \tan \kappa_{c}\right. \\
& \left.\quad-\frac{\varrho_{c a}^{2}+\varrho_{a c}^{2}}{2 \varrho_{c a} \varrho_{a c}} \tan \kappa_{c} \tan \kappa_{a}\right),
\end{aligned}
$$

where $k_{x}$ is the Bloch wave vector, $k_{z}$ the propagation wave vector, $t_{a}, t_{b}$, and $t_{c}$ are the thicknesses of the layers, $\Lambda=t_{a}+$ $t_{b}+t_{c}$ is the period of the supercell, $\sigma=\cos \kappa_{a} \cos \kappa_{b} \cos \kappa_{c}$, and $k_{j}=\sqrt{(\omega / c)^{2} \varepsilon_{j} \mu_{j}-k_{z}^{2}}$, with $j=a, b, c$, where $a, b$, and $c$ stand for graphene, silicon, and silver, respectively. For convenience, we here define $\varrho_{i j} \equiv \kappa_{i} \tau_{j}, \kappa_{i} \equiv k_{i} t_{i}$, and $\tau_{i} \equiv \varepsilon_{i} t_{i}$. By fixing frequency $\omega$ in Eq. (1), dependence $k_{z}=k_{z}\left(k_{x}\right)$ determines the spatial dispersion relation for the particular frequency.

Tunability and robustness of the Dirac points. A peculiar property of the band structure of such GPSs is that, if it possesses two transmission bands, then the bands intersect at the center of the Brillouin zone, $k_{x}=0$, giving rise to the photonic DP [see Fig. 1(b)]. For such a two-band configuration to occur, the thickness of the dielectric and metallic layers should be larger than certain critical values. As mentioned above, a DP in our photonic structure exists provided that the real part of the average permittivity of the superlattice vanishes, namely,

$$
\operatorname{Re}(\bar{\varepsilon})=\frac{1}{\Lambda} \operatorname{Re}\left(\tau_{g}+\tau_{m}+\tau_{d}\right)=0 .
$$

DPs are well known in solid-state physics, being at the origin of many remarkable properties of recently discovered materials, such as graphene and topological insulators. In this case they are singular points in the electron energy-band structure, and the aforementioned similarities between electron and photon wave dynamics suggest that DPs can also exist in the photonic band structure of certain periodic structures, one such example being the GPS presented in Fig. 1(a). As we demonstrate in this study, the graphene component of the

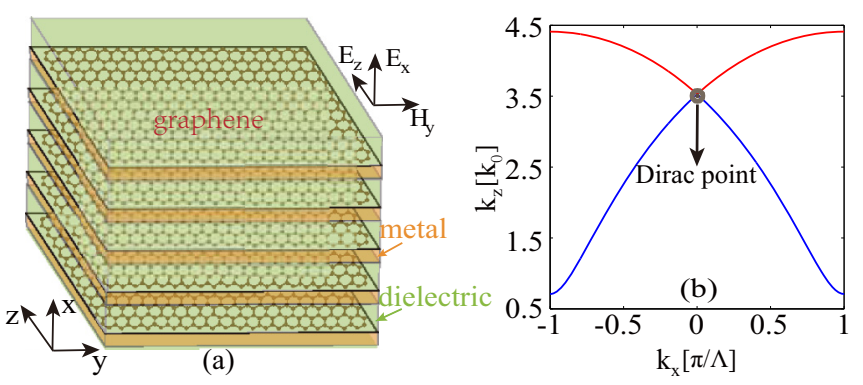

FIG. 1. (Color online) (a) A schematic of the graphene photonic superlattice: The unit cell consists of a graphene-metal-dielectric trilayer configuration stacked along the $x$ direction. The incident transverse magnetic (TM)-polarized beam propagates along the $z$ axis, its nonvanishing field components being $E_{x}, E_{z}$, and $H_{y}$. (b) The photonic band structure calculated in the zero-averaged-permittivity case, featuring the $1 \mathrm{D}$ Dirac point. The structure parameters are $\varepsilon_{g}=-14.48, \varepsilon_{\mathrm{Si}}=12.25, \varepsilon_{m}=-924.05, t_{g}=0.5 \mathrm{~nm}, t_{\mathrm{Si}}=$ $604 \mathrm{~nm}, t_{m}=8 \mathrm{~nm}$, and $\lambda=4.2 \mu \mathrm{m}$.
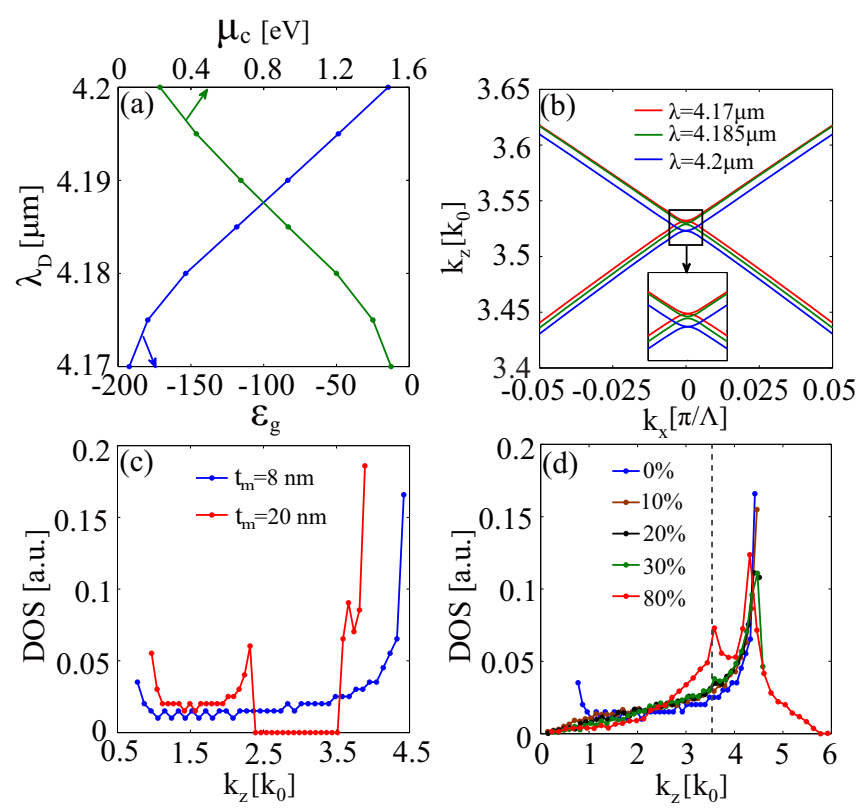

FIG. 2. (Color online) (a) The dependence of the wavelength of the Dirac point on the graphene's chemical potential and permittivity. (b) The photonic band structure calculated for different wavelengths. Parameters are the same as in Fig. 1. (c) The density of states of a 50-period GPS possessing a Dirac point (at $t_{m}=8 \mathrm{~nm}$ ) or a Bragg gap (at $t_{m}=20 \mathrm{~nm}$ ). (d) The density of states of the GPS in (c) that possesses a Dirac point, calculated for different disorder levels.

present photonic superlattice allows one to tune the wavelength of the photonic DPs by simply varying the chemical potential of electrons in graphene, $\mu_{\mathrm{c}}$. More specifically, changing $\mu_{\mathrm{c}}$ by external means (e.g., gate voltage, chemical doping, etc.), one varies the permittivity of graphene and therefore one can tune the wavelength $\lambda_{D}$ at which condition $\operatorname{Re}\left[\bar{\varepsilon}\left(\lambda_{D}\right)\right]=0$ holds. These ideas are illustrated in Fig. 2(a), where we plot the dependence of the wavelength of the DP on $\mu_{\mathrm{c}}$. Note that a spectral shift of more than $30 \mathrm{~nm}$ can be achieved changing $\mu_{\mathrm{c}}$ by $1.5 \mathrm{eV}$. The variation of the corresponding band structure is presented in Fig. 2(b).

Similar to zero- $\bar{n}$ band gaps, which are formed when the spatial average of the refractive index of a Bragg grating vanishes, DPs in our photonic structure are particularly robust against disorder added to the system. This behavior is expected because structural disorder weakly affects the spatial average of the permittivity, hence its vanishing, which determines the existence of DPs, persists in the presence of disorder as well. To illustrate this phenomenon, we present in Figs. 2(c) and 2(d) the density of states (DOS) of periodic and disordered GPSs, respectively. In the calculations, we used two superlattices, one selected so as to possess a DP (at $t_{m}=8 \mathrm{~nm}$ ), whereas the other one is a regular superlattice featuring a Bragg gap, at $t_{m}=20 \mathrm{~nm}$. The DOS of these two superlattices is extracted from $d k_{z} / d k_{x}$ [see the results in Fig. 2(c)]. As expected, while the DOS for the superlattice featuring a Bragg gap exhibits a conspicuous wide region of forbidden states (DOS $=0$ ), the superlattice possessing a DP shows nonvanishing DOS in the whole spectrum. The robustness of the DP against the structural disorder is confirmed in Fig. 2(d), where we 
display DOS for the disordered superlattice at several different levels of the disorder. It is introduced by assuming random fluctuations of the metallic-layer thickness around its average value, namely, the $n$th thickness is $t_{m}^{n}=t_{m 0}+\delta_{n}$, where $t_{m 0}$ is the average thickness (we choose $t_{m 0}=8 \mathrm{~nm}$ here), and $\delta_{n}$ is a random perturbation. We assume $\delta_{n}$ to be uniformly distributed in the interval of $[-\delta, \delta]$, hence the disorder level may be characterized by $\Delta \equiv \delta / t_{m 0}$. The DOSs in Fig. 2(d) clearly demonstrate that, even when the disorder level is increased to $80 \%$, the DP is preserved, appearing as a spectral peak in the DOS, at $\lambda_{D} \simeq 4.2 \mu \mathrm{m}$.

Real-time tunability of the band structure of the GPS provides an effective way to control the excitation and propagation of optical beams. Figures 3(a) and 3(b) show that, when one varies $\mu_{\mathrm{c}}$ from 0.23 to $1.5 \mathrm{eV}$, the band structure transforms from a gapless one, which possesses a DP, to a gapped structure, this effect being accompanied by a dramatic change in the beam-propagation dynamics. At the DP, a normally incident Gaussian beam splits into two, as the upper- and lower-band excitations are not resolved, due to the fact that the two bands have identical slopes near the
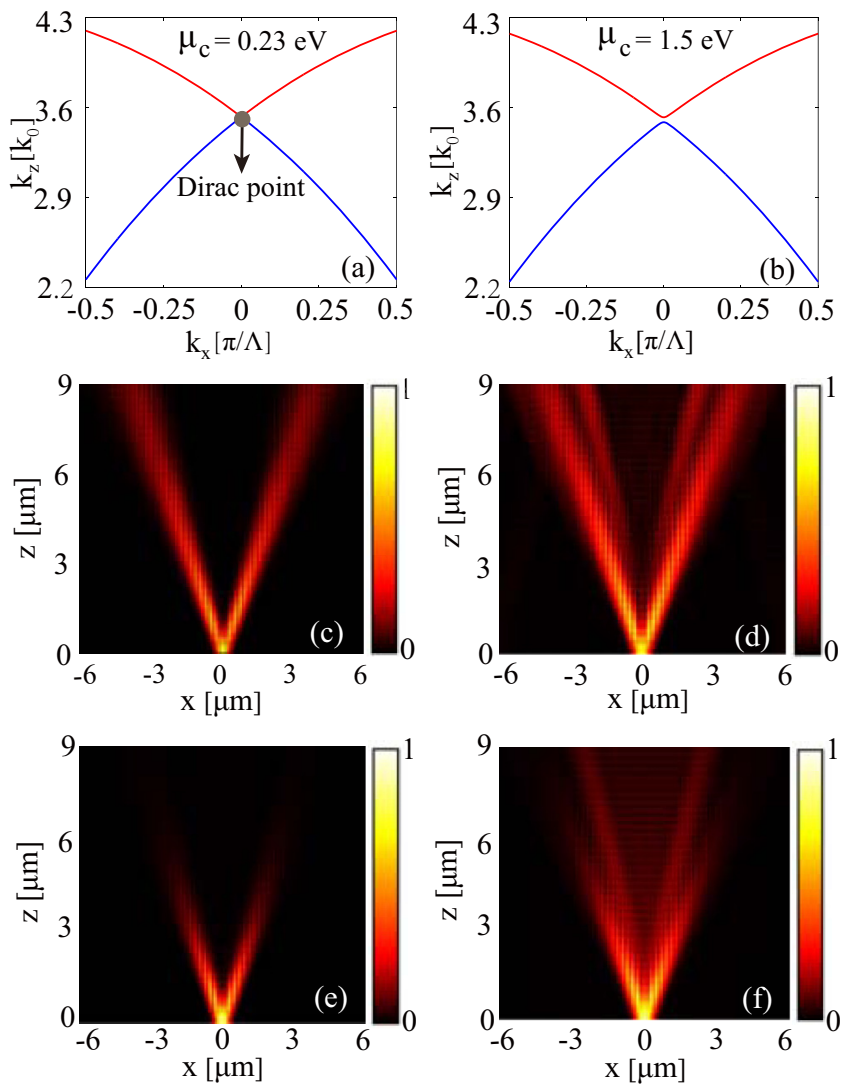

FIG. 3. (Color online) (a), (b) Formation of a band gap at the Dirac point with $\lambda_{D}=4.2 \mu \mathrm{m}$ by varying the graphene's Fermi level. In (a) and (c), permittivities of the layers are the same as in Fig. 1. In (b) and (d), $\varepsilon_{g}=-195.02$. The real part of the permittivities in (e) and (f) are the same as those in (c) and (d), respectively, whereas in (e) $\varepsilon_{g}^{\prime \prime}=1.28 i, \varepsilon_{m}^{\prime \prime}=56.37 i$ and in (f) $\varepsilon_{g}^{\prime \prime}=4.95 i, \varepsilon_{m}^{\prime \prime}=56.37 i$. In all cases, the thicknesses of the layers are the same as in Fig. 1, and the input conditions select a TM-polarized Gaussian beam with profile $E_{x}(x)=\exp \left(-x^{2} / w^{2}\right)$, where $w=2 \lambda_{D}$.
DP [see Fig. 3(c)]. By contrast, when a gap opens the output pattern exhibits four beams, due to the difference in the slopes of the upper and lower bands near $k_{x}=0$ [as per Fig. 3(d)]. Taking losses in graphene and metallic layers into account yields similar patterns of the output optical field [Figs. 3(e) and 3(f)]; however, as expected, the output beams now decay in the course of the propagation. In Figs. 3(e) and 3(f) we used the scattering time, $\tau=90 \mathrm{fs}$, which was measured in an ionic-liquid gating configuration [28], the achieved Fermi level being as large as $2 \mathrm{eV}[29,30]$.

Optically tunable Dirac points. In addition to electrical means, the wavelength at the DPs can be tuned by means of the intrinsic optical nonlinearity of graphene, or of the dielectric and metallic layers as well. Such nonlinearityinduced tunability of the DP is possible in our superlattices because, as explained above, the DPs form when the real part of the average permittivity is zero. Thus, nonlinear optical effects that change the refractive index can be used to tune the permittivity in or out of the zero-average state, thus providing all-optical means to control the formation of DPs. The optical nonlinearity of graphene is expected to be particularly strong, due to its unusually large third-order nonlinear susceptibility [31], as well as the tightly localized optical field supported by graphene sheets.

Figure 4 illustrates the creation and elimination of a DP under the action of the optical Kerr effect, induced in graphene and the neighboring silicon layer. At low optical powers, starting with a superlattice for which $\bar{\varepsilon}(\lambda)<0$, the two transmission bands are separated by a gap, as seen in Fig. 4(a). When the input power increases, both graphene and
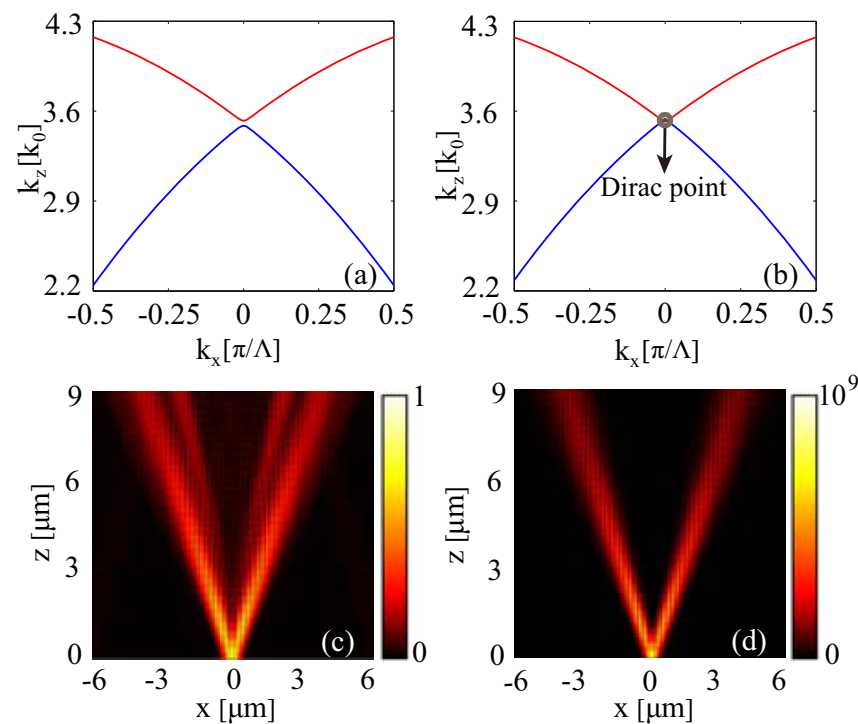

FIG. 4. (Color online) (a) The linear band structure, calculated for the same parameters as in Fig. 1, except $\varepsilon_{g}=-195.02(\bar{\varepsilon}=$ -0.15 ). (b) The nonlinear band structure, where the Bloch mode of the upper band at $k_{x}=0$ is used to induce the nonlinear change of permittivities, $\delta \varepsilon_{\mathrm{Si}}=0.07$ and $\delta \varepsilon_{g}=0.46$. In (c) and (d), the input condition is a TM-polarized Gaussian beam with $E_{x}(x)=$ $A \exp \left(-x^{2} / w^{2}\right)$, with $w=2 \Lambda$, and $A=1 \mathrm{~V} / \mathrm{m}$ in (c) or $A=4.13 \times$ $10^{7} \mathrm{~V} / \mathrm{m}$ in (d). The Kerr coefficients are $n_{2, \mathrm{Si}}=1.86 \times 10^{-20} \mathrm{~m}^{2} / \mathrm{V}^{2}$ and $n_{2, g}=4.09 \times 10^{-17} \mathrm{~m}^{2} / \mathrm{V}^{2}$. 

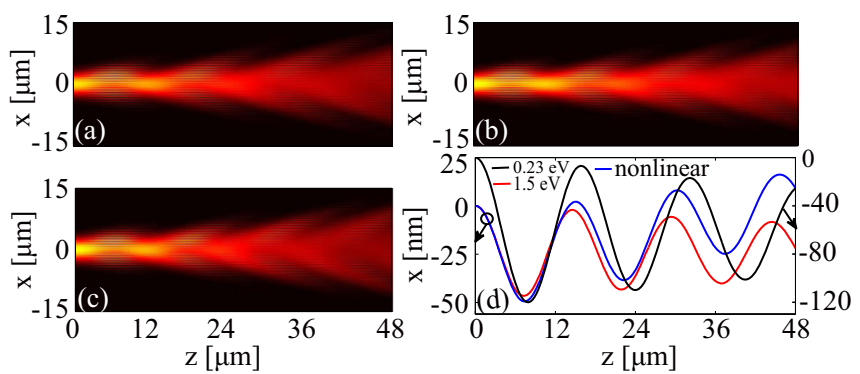

FIG. 5. (Color online) Beam propagation in the linear regime: (a) $\mu_{\mathrm{c}}=0.23 \mathrm{eV}$, (b) $\mu_{\mathrm{c}}=1.5 \mathrm{eV}$. (c) The propagation in the nonlinear regime, with $\mu_{\mathrm{c}}=1.5 \mathrm{eV}$. (d) Dependence of the center of mass of the beam on $z$. The input condition is a TM-polarized Gaussian beam with $E_{x}(x)=A \exp \left(-x^{2} / w^{2}\right)$, with $w=12 \Lambda$. In (c) $A=1.5 \times 10^{7} \mathrm{~V} / \mathrm{m}$. The thicknesses of the layers are $t_{g}=0.5 \mathrm{~nm}$, $t_{\mathrm{Si}}=604 \mathrm{~nm}$, and $t_{m}=10 \mathrm{~nm}$, whereas $n_{2, \mathrm{Si}}=1.86 \times 10^{-20} \mathrm{~m}^{2} / \mathrm{V}^{2}$ and $n_{2, g}=4.09 \times 10^{-17} \mathrm{~m}^{2} / \mathrm{V}^{2}$.

silicon are affected by the Kerr nonlinearity, their permittivities varying by $\delta \varepsilon_{g}>0$ and $\delta \varepsilon_{\mathrm{Si}}>0$, respectively. As a result, the average permittivity of the structure $\bar{\varepsilon}$ gradually increases and the corresponding band structure is modified as well. At some particular value of the optical power, $\bar{\varepsilon}$ becomes equal to zero, so that, as shown in Fig. 4(b), the photonic gap closes and a DP reemerges. For the particular case illustrated in Fig. $4, \delta \varepsilon_{\mathrm{Si}}=0.07$ and $\delta \varepsilon_{g}=0.46$. The propagation of a TM-polarized Gaussian beam, both in the linear and nonlinear regimes, is presented in Figs. 4(c) and 4(d), respectively. One can see that, when the power of the input beam increases, the beam dynamics changes from the typical discrete diffraction to $1 \mathrm{D}$ conical diffraction, which is a manifestation of the formation of the DP in the latter case.

Tunable Zitterbewegung effect. Finally, we point out that the control of the DP and band structure of GPSs suggests a convenient way to implement, in the classical-optics context, a series of intriguing quantum-mechanical phenomena. As a relevant example, here we consider the $\mathrm{ZB}$ effect, which, in the relativistic quantum mechanics, refers to rapid oscillations (trembling) of a moving electron around its average position. The $\mathrm{ZB}$ of electrons arises because of the interference of particle and antiparticle components of the wave function. The electron ZB oscillations have extremely high frequencies, of about $10^{21} \mathrm{~Hz}$, therefore this phenomenon is not accessible to experimental observations. Recently, optical analogs of the ZB were studied in 1D [22-26] and 2D [21] photonic crystals. Here we demonstrate that GPSs are highly suitable physical systems to study this effect. To this end, we show in Figs. 5(a) and 5(b) the beam propagation at two different values of the chemical potential, $\mu_{\mathrm{c}}=0.23$ and $1.5 \mathrm{eV}$, respectively. Note that in both cases the GPS has gaps in the wave-vector space, the gap at $\mu_{\mathrm{c}}=1.5 \mathrm{eV}$ being wider. In the latter case, the $\mathrm{ZB}$ has a smaller oscillation amplitude and a larger frequency, which is consistent with the findings reported in Ref. [24]. This behavior is more clearly observed in Fig. 5(d), where we plot the evolution of the beam center, $x_{c} \equiv \int_{-\infty}^{+\infty}|\mathbf{E}(x)|^{2} x d x / \int_{-\infty}^{+\infty}|\mathbf{E}(x)|^{2} d x$.

Still more important, GPSs provide a reliable platform to implement "nonlinear quantum mechanics," as shown in Fig. 5(c), where we examine the ZB dynamics affected by the self-focusing nonlinearity, associated with the graphene and silicon layers. The figure shows that, while the ZB amplitude and frequency remain almost unaltered, the self-focusing nonlinearity leads to a spatial walk-off of the $\mathrm{ZB}$, viz., monotonic spatial drift of the beam's center in one direction, in the course of its propagation. Note that, as the thickness of the layers of the GPSs can be scaled so that the real part of its averaged permittivity remains equal to zero, one can easily extend the ZB effect, and the emulation of other DP-related quantum phenomena, into the $\mathrm{THz}$ and other spectral ranges, different from the standard optical one.

Conclusion. We have proposed graphene-based photonic superlattices which should provide a highly effective and robust approach for the control of photonic beams at the nanoscale. By taking advantage of the strong dependence of the graphene permittivity on the chemical potential, it is demonstrated that, due to the peculiar topology of their photonic band structure, specially designed photonic superlattices, incorporating graphene sheets interlaid with metallic and dielectric layers, feature large electrical and optical tunability. Implications of our findings to applications and studies of fundamental effects, such as Zitterbewegung, are also proposed.

Acknowledgments. The work of H.D. and F.Y. was supported by Innovation Program of Shanghai Municipal Education Commission (Grant No. 13ZZ022) and the National Natural Science Foundation of China (Grants No. 11104181 and No. 61475101). The work of N.C.P. was supported by the Engineering and Physical Sciences Research Council, Grant No. EP/J018473/1. N.C.P. acknowledges the hospitality of the Department of Physics and Astronomy of Shanghai Jiao Tong University during a visit to which this paper was written. B.A.M. appreciates a partial support through Grant No. B12024 from "Program of introducing talents of discipline to universities" (China).
[1] I. I. Smolyaninov, A. V. Zayats, A. Gungor, and C. C. Davis, Phys. Rev. Lett. 88, 187402 (2002).

[2] W. Cai, A. P. Vasudev, and M. L. Brongersma, Science 333, 1720 (2011).

[3] N. C. Panoiu and R. M. Osgood, Nano Lett. 4, 2427 (2004).

[4] J. Elliott, I. I. Smolyaninov, N. I. Zheludev, and A. V. Zayats, Opt. Lett. 29, 1414 (2004).
[5] E. J. Osley, C. G. Biris, P. G. Thompson, R. R. F. Jahromi, P. A. Warburton, and N. C. Panoiu, Phys. Rev. Lett. 110, 087402 (2013).

[6] I. M. Pryce, K. Aydin, Y. A. Kelaita, R. M. Briggs, and H. A. Atwater, Nano Lett. 10, 4222 (2010).

[7] D. H. Werner, D.-H. Kwon, I.-C. Khoo, I.-C. Kildishev, A. V. Shalaev, and M. Vladimir, Opt. Express 15, 3342 (2007). 
[8] H.-T. Chen, W. J. Padilla, J. M. O. Zide, A. C. Gossard, A. J. Taylor, and R. D. Averitt, Nature (London) 444, 597 (2006).

[9] F. Bonaccorso, Z. Sun, T. Hasan, and A. C. Ferrari, Nat. Photonics 4, 611 (2010).

[10] J. Chen, M. Badioli, P. A. Gonzalez, S. Thongrattanasiri, F. Huth, J. Osmond, M. Spasenovic, A. Centeno, A. Pesquera, P. Godignon, A. Z. Elorza, N. Camara, F. J. G. de Abajo, R. Hillenbrand, and F. H. L. Koppens, Nature (London) 487, 77 (2012).

[11] Z. Fei, A. S. Rodin, G. O. Andreev, W. Bao, A. S. McLeod, M. Wagner, L. M. Zhang, Z. Zhao, M. Thiemens, G. Dominguez, M. M. Fogler, A. H. Castro Neto, C. N. Lau, F. Keilmann, and D. N. Basov, Nature (London) 487, 82 (2012).

[12] M. Jablan, H. Buljan, and M. Soljacic, Phys. Rev. B 80, 245435 (2009).

[13] F. H. L. Koppens, D. E. Chang, and F. J. G. de Abajo, Nano Lett. 11, 3370 (2011)

[14] H. Yan, X. Li, B. Chandra, G. Tulevski, Y. Wu, M. Freitag, W. Zhu, P. Avouris, and F. Xia, Nat. Nanotechnol. 7, 330 (2012).

[15] H. Yan, F. Xia, Z. Li, and P. Avouris, New J. Phys. 14, 125001 (2012).

[16] Y. Yao, M. A. Kats, P. Genevet, N. Yu, Y. Song, J. Kong, and F. Capasso, Nano Lett. 13, 1257 (2013).

[17] Z. Fang, Y. Wang, A. E. Schlather, Z. Liu, P. M. Ajayan, F. J. G. de Abajo, P. Nordlander, X. Zhu, and N. J. Halas, Nano Lett. 14, 299 (2014).
[18] Y. P. Bliokh, V. Freilikher, and F. Nori, Phys. Rev. B 87, 245134 (2013).

[19] A. V. Shytov, M. S. Rudner, and L. S. Levitov, Phys. Rev. Lett. 101, 156804 (2008).

[20] A. F. Young and P. Kim, Nat. Phys. 5, 222 (2009).

[21] X. Zhang, Phys. Rev. Lett. 100, 113903 (2008).

[22] S. Longhi, Opt. Lett. 35, 235 (2010).

[23] S. H. Nam, A. J. Taylor, and A. Efimov, Opt. Express 18, 10120 (2010).

[24] F. Dreisow, M. Heinrich, R. Keil, A. Tunnermann, S. Nolte, S. Longhi, and A. Szameit, Phys. Rev. Lett. 105, 143902 (2010).

[25] Q. Liang, Y. Yan, and J. Dong, Opt. Lett. 36, 2513 (2011).

[26] S. Ding and G. P. Wang, J. Opt. Soc. Am. B 31, 603 (2014).

[27] See Supplemental Material at http://link.aps.org/supplemental/ 10.1103/PhysRevB.91.201402 for (1) a description of the physical models of permittivity of noble metals and graphene, (2) the derivation of the dispersion relation of the Bloch modes of graphene superlattices, (3) a discussion of the influence of metallic and graphene optical losses on the Zitterbewegung effect, and (4) a discussion of the effective linear and nonlinear susceptibilities of graphene.

[28] P. Tassin, T. Koschny, and C. M. Soukoulis, Science 341, 620 (2013).

[29] J. Ye, M. F. Craciun, M. Koshino, S. Russo, S. Inoue, H. Yuan, H. Shimotani, A. F. Morpurgo, and Y. Iwasa, Proc. Natl. Acad. Sci. USA 108, 13002 (2011).

[30] D. K. Efetov and P. Kim, Phys. Rev. Lett. 105, 256805 (2010).

[31] E. Hendry, P. J. Hale, J. Moger, A. K. Savchenko, and S. A. Mikhailov, Phys. Rev. Lett. 105, 097401 (2010). 


\title{
Optically and electrically tunable Dirac points and Zitterbewegung in graphene-based photonic superlattices
}

\author{
Hanying Deng, ${ }^{1,2}$ Fangwei Ye, ${ }^{1,2, *}$ Boris A. Malomed ${ }^{3}$ Xianfeng Chen, ${ }^{1,2}$ and Nicolae C. Panoiu ${ }^{4}$ \\ ${ }^{1}$ Department of Physics and Astronomy, Shanghai Jiao Tong University, Shanghai 200240, China \\ ${ }^{2}$ Key Laboratory for Laser Plasma (Ministry of Education), \\ IFSA Collaborative Innovation Center, Shanghai Jiao Tong University, Shanghai 200204, China \\ ${ }^{3}$ Department of Physical Electronics, School of Electrical Engineering, \\ Faculty of Engineering, Tel Aviv University, Tel Aviv 69978, Israel \\ ${ }^{4}$ Department of Electronic and Electrical Engineering, University College London, \\ Torrington Place, London WC1E7JE, United Kingdom
}

\section{S1. Physical models for the electric permittivity of metal and graphene}

For the metallic layers of our photonic superlattice we use the Drude model for the complex, frequency-dependent electric permittivity of the metal:

$$
\varepsilon_{m}=1-\frac{\omega_{p}^{2}}{\omega(\omega+i \nu)}
$$

where $\omega_{p}$ and $\nu$ are the plasma and damping frequencies, respectively. In the paper, the metallic layers are assumed to be made of silver, with $\omega_{p}=13.7 \times 10^{15} \mathrm{rad} / \mathrm{s}$ and $\nu=2.7 \times 10^{13} \mathrm{rad} / \mathrm{s}$ [? ]. For graphene, we use the Kubo's formula to model the surface conductivity, $\sigma_{g}$, including the contributions of both the interband and intraband transitions $[?$ ],

$$
\sigma_{s}\left(\omega, \mu_{c}, \tau, T\right)=-\frac{i e^{2}\left(\omega+i \tau^{-1}\right)}{\pi \hbar^{2}}\left\{\int_{-\infty}^{+\infty} \frac{\partial f(\epsilon)}{\partial \epsilon} \frac{|\epsilon| d \epsilon}{\left(\omega+i \tau^{-1}\right)^{2}}-\int_{0}^{+\infty}\left[\frac{\partial f(-\epsilon)}{\partial \epsilon}-\frac{\partial f(\epsilon)}{\partial \epsilon}\right] \frac{d \epsilon}{\left(\omega+i \tau^{-1}\right)^{2}-4(\epsilon / \hbar)^{2}}\right\}
$$

Here, $f(\epsilon)=1 /\left\{1+\exp \left[\left(\epsilon-\mu_{c}\right) /\left(k_{\mathrm{B}} T\right)\right]\right\}$ is the Fermi-Dirac distribution, with $k_{\mathrm{B}}$ being the Boltzmann constant, $\epsilon$ the energy, $\mu_{c}$ the chemical potential, $T$ the temperature, $e$ the electron charge, $\hbar$ the reduced Planck's constant, and $\tau$ the momentum relaxation time (inverse of the electron-phonon scattering rate). A carrier density as large as $n \approx 2 \times 10^{14} \mathrm{~cm}^{-2}$ has been achieved experimentally with an ionic liquid gating [? ], which corresponds to a Fermi level $\mu_{c}=1.65 \mathrm{eV}\left(\mu_{c}=\hbar V_{\mathrm{F}} \sqrt{\pi n}\right.$, see [? ? ]). We also note that $n \approx 4 \times 10^{14} \mathrm{~cm}^{-2}$ has been realized with an electrolytic gate [?], which means $\mu_{c}=2.33 \mathrm{eV}$. For such gating schemes, the typical scattering time is of the order of $100 \mathrm{fs}$ [? ]. In the simulations pertaining to Fig. 3(e) and 3(f) of the main text, as well as Fig. 2 of the supporting materials, we used in Eq. (2) the experimentally measured value, $\tau=90 \mathrm{fs}[?]$.

The first term in Eq. (2) corresponds to intraband electron-photon scattering processes, and can be evaluated to

$$
\sigma_{\text {intra }}=i \frac{e^{2} k_{B} T}{\pi \hbar^{2}\left(\omega+i \tau^{-1}\right)}\left[\frac{\mu_{\mathrm{c}}}{k_{B} T}+2 \ln \left(e^{-\frac{\mu_{c}}{k_{\mathrm{B}} T}}+1\right)\right]
$$

whereas the second term corresponds to direct interband electron transitions and, for $\hbar \omega,\left|\mu_{c}\right| \gg k_{\mathrm{B}} T$, it can be approximated to

$$
\sigma_{\text {inter }}=i \frac{e^{2}}{4 \pi \hbar} \ln \left[\frac{2\left|\mu_{c}\right|-\hbar\left(\omega+i \tau^{-1}\right)}{2\left|\mu_{c}\right|+\hbar\left(\omega+i \tau^{-1}\right)}\right]
$$

\section{S2. Derivation of Eq. (1) in the Main Text}

We use the transfer matrix method (TMM) to obtain the photonic bandstrcture of the superlattice. Thus, the electromagnetic field at two positions $x$ and $x+\Delta x$ in the same layer is related via a transfer matrix [? ? ]:

$$
M_{j}(\Delta x, \omega)=\left(\begin{array}{cc}
\cos \left(k_{j} \triangle x\right) & \frac{i}{q_{j}} \sin \left(k_{j} \triangle x\right) \\
i q_{j} \sin \left(k_{j} \triangle x\right) & \cos \left(k_{j} \triangle x\right)
\end{array}\right),
$$

\footnotetext{
* fangweiye@sjtu.edu.cn
} 


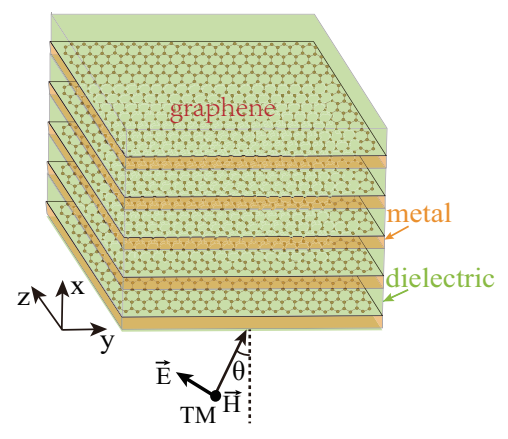

FIG. 1. A schematic of the graphene photonic superlattice: the unit cell consists of a graphene-metal-dielectric tri-layer configuration stacked along the $x$ direction. The incident TM-polarized beam propagates along the $z$-axis, its non-vanishing field components being $E_{x}, E_{z}$, and $H_{y}$.

where $k_{j}=\sqrt{(\omega / c)^{2} \varepsilon_{j} \mu_{j}-k_{z}^{2}}, j=a, b, c$. Here, $a, b$, and $c$ stand for graphene, silicon, and silver, respectively. For a TM-polarized wave, $q_{j}=\left[\mu_{j} /\left(\varepsilon_{j}-\sin ^{2} \theta / \mu_{j}\right)\right]^{1 / 2}$. In Figs. S1, we show the structure of the superlattice we consider.

According to the Bloch theorem, the electric and magnetic components of an electromagnetic mode in layer $N$ and $N+1$, with Bloch wavevector, $k_{x}$, are related to each other through,

$$
\left(\begin{array}{c}
E_{N} \\
H_{N}
\end{array}\right)=e^{i k_{x} \Lambda}\left(\begin{array}{c}
E_{N-1} \\
H_{N-1}
\end{array}\right)
$$

On the other hand, the TMM leads to:

$$
\left(\begin{array}{c}
E_{N} \\
H_{N}
\end{array}\right)=M_{a} M_{b} M_{c}\left(\begin{array}{c}
E_{N+1} \\
H_{N+1}
\end{array}\right) \equiv\left(\begin{array}{ll}
m_{11} & m_{12} \\
m_{21} & m_{22}
\end{array}\right)\left(\begin{array}{c}
E_{N+1} \\
H_{N+1}
\end{array}\right)
$$

Combining Eqs. (7) and (8) yields:

$$
\left(\begin{array}{ll}
m_{11} & m_{12} \\
m_{21} & m_{22}
\end{array}\right)\left(\begin{array}{l}
E_{N} \\
H_{N}
\end{array}\right)=e^{i k_{x} \Lambda}\left(\begin{array}{c}
E_{N} \\
H_{N}
\end{array}\right),
$$

so that the factor $e^{i k_{x} \Lambda}$ is the eigenvalue of the transfer matrix $M=M_{a} M_{b} M_{c}$. This conclusion can be expressed as

$$
e^{i k_{x} \Lambda}=\frac{1}{2}\left(m_{11}+m_{22}\right) \pm\left[\frac{1}{4}\left(m_{11}+m_{22}\right)^{2}-1\right]^{\frac{1}{2}} .
$$

From this equation one can easily derive the dispersion relation, $k_{x}=k_{x}\left(k_{z}, \omega\right)$,

$$
k_{x}\left(k_{z}, \omega\right)=\frac{1}{\Lambda} \arccos \left[\frac{1}{2}\left(m_{11}+m_{22}\right)\right] .
$$

By substituting the elements of the matrix $M$, this relation yields,

$$
\begin{aligned}
\cos \left(k_{x} \Lambda\right)= & \cos \left(k_{a} t_{a}\right) \cos \left(k_{b} t_{b}\right) \cos \left(k_{c} t_{c}\right)-\frac{1}{2}\left(\frac{\varepsilon_{a} k_{b}}{\varepsilon_{b} k_{a}}+\frac{\varepsilon_{b} k_{a}}{\varepsilon_{a} k_{b}}\right) \sin \left(k_{a} t_{a}\right) \sin \left(k_{b} t_{b}\right) \cos \left(k_{c} t_{c}\right) \\
& -\frac{1}{2}\left(\frac{\varepsilon_{a} k_{c}}{\varepsilon_{c} k_{a}}+\frac{\varepsilon_{c} k_{a}}{\varepsilon_{a} k_{c}}\right) \sin \left(k_{a} t_{a}\right) \cos \left(k_{b} t_{b}\right) \sin \left(k_{c} t_{c}\right)-\frac{1}{2}\left(\frac{\varepsilon_{c} k_{b}}{\varepsilon_{b} k_{c}}+\frac{\varepsilon_{b} k_{c}}{\varepsilon_{c} k_{b}}\right) \cos \left(k_{a} t_{a}\right) \sin \left(k_{b} t_{b}\right) \sin \left(k_{c} t_{c}\right) .
\end{aligned}
$$

Finally, Eq. (12) can be simplified to

$$
\cos \left(k_{x} \Lambda\right)=\sigma\left(1-\frac{\varrho_{a b}^{2} \varrho_{b a}^{2}}{2 \varrho_{a b} \varrho_{b a}} \tan \kappa_{a} \tan \kappa_{b}-\frac{\varrho_{b c}^{2}+\varrho_{c b}^{2}}{2 \varrho_{b c} \varrho_{c b}} \tan \kappa_{b} \tan \kappa_{c}-\frac{\varrho_{c a}^{2}+\varrho_{a c}^{2}}{2 \varrho_{c a} \varrho_{a c}} \tan \kappa_{c} \tan \kappa_{a}\right),
$$

where $\sigma=\cos \kappa_{a} \cos \kappa_{b} \cos \kappa_{c}$. We have also defined $\varrho_{i j}=\kappa_{i} \tau_{j}, \kappa_{i}=k_{i} t_{i}$, and $\tau_{i}=\varepsilon_{i} t_{i}$, where $i, j=a, b, c$. 

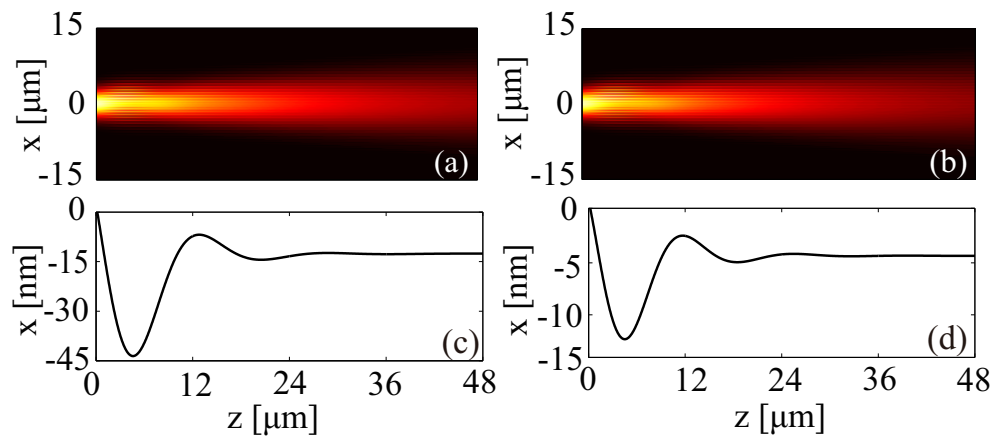

FIG. 2. Beam propagation in the presence of optical losses in the metal and graphene layers and the dependence on $z$ of the center of mass of the optical beam. In (a) and (c), $\mu_{c}=0.23 \mathrm{eV}$ whereas in (b) and (d) $\mu_{c}=1.5 \mathrm{eV}$. The real part of the permittivities in (a), (c) and (b), (d) are the same as those in Figs. 5(a) and 5(b) in the Main Text, respectively, whereas the imaginary part of the permittivities in panels (a) and (c) are $\varepsilon_{g}^{\prime \prime}=1.28 i, \varepsilon_{m}^{\prime \prime}=56.37 i$, whereas in panels (b) and (d) they are $\varepsilon_{m}^{\prime \prime}=56.37 i$ and $\varepsilon_{g}^{\prime \prime}=4.95 i$.

\section{S3. The influence of metallic and graphene optical losses on the Zitterbewegung effect}

If we take into account in our numerical simulations the influence of optical losses in the metallic and graphene components of the superlattice, the Zitterbewegung (ZB) effect illustrated in Figs. 5(a) and 5(b) in the Main Text will be modified as shown in Figs. 2(a) and 2(b), respectively. It should be noted that the ZB effect still manifests in the beam dynamics, especially during the initial stages of the beam propagation [as per Figs. 2(c) and 2(d)].

\section{S4. The linear and nonlinear permittivity of graphene}

For the sake of convenience in numerical simulations, graphene layers have been approximated by finite-thickness layers with the effective dielectric constant given by [? ? ],

$$
\varepsilon_{g, t o t}=1+\frac{i \sigma_{g, t o t} \eta_{0}}{k_{0} t_{g}}=1+\frac{i \eta_{0}}{k_{0} t_{g}}\left(\sigma_{s}+\sigma_{3}\left|\mathrm{E}_{\|}\right|^{2}\right)=\varepsilon_{g}+\chi_{g}^{(3)}\left|\mathrm{E}_{\|}\right|^{2}
$$

where $\eta_{0}$ is the vacuum impedance $\left(\eta_{0} \approx 377 \Omega\right), t_{g}$ is the thickness of graphene $\left(t_{g}=0.5\right.$ nm used in our simulations $)$, $\mathrm{E}_{\|}$is the in-plane component of electric field, and $k_{0}=2 \pi / \lambda$ is the wavenumber in vacuum of light with wavelength, $\lambda$. The nonlinear contribution to the surface conductivity of the graphene is given by, $\sigma_{3}\left|\mathrm{E}_{\|}\right|^{2}$, where $\sigma_{3}$ is the nonlinear conductivity coefficient, expressed as [? ? ]

$$
\sigma_{3}=-i \frac{3}{32} \frac{e^{4} v_{F}^{2}}{\pi \hbar^{2} \mu_{c} \omega^{3}}
$$

In this equation, $v_{F} \approx \mathrm{c} / 300$ is the Fermi velocity. Equivalently, one can define a third-order effective nonlinear susceptibility, $\chi_{g}^{(3)}=\frac{i \sigma_{3} \eta_{0}}{k_{0} t_{g}}$. If we assume $\mu_{c}=1.5 \mathrm{eV}$ and $\lambda=4.2 \mu \mathrm{m}$, as we used in the main text, one finds $\chi_{g}^{(3)}=4.09 \times 10^{-17} \mathrm{~m}^{2} / \mathrm{V}^{2}$.

[1] M. Ordal, R. Bell, R. Alexander, Jr, L. Long, and M. Querry, Appl. Opt. 24, 4493 (1985).

[2] L. Falkovsky and S. Pershoguba, Phys. Rev. B 76, 153410 (2007).

[3] J. Ye, M. F. Craciun, M. Koshino, S. Russo, S. Inoue, H. Yuan, H. Shimotani, A. F. Morpurgo, and Y. Iwasa, Proc. Natl. Acad. Sci. 108, 13002 (2011).

[4] K. S. Novoselov, A. K Geim et al., Nature (London) 438, 197 (2005).

[5] Y. B. Zhang, Y. W. Tan, H. L. Stormer, and P. Kim, Nature (London) 438, 201 (2005).

[6] D. K. Efetov and P. Kim, Phys. Rev. Lett. 105, 256805 (2010).

[7] P. Tassin, T. Koschny, and C. M. Soukoulis, Science 341, 620 (2013).

[8] N. Liu, S. Y. Zhu, H. Chen, and X. Wu, Phys. Rev. E 65, 046607 (2002).

[9] P. Yeh, A. Yariv, and C. S. Hong, J. Opt. Soc. Am. 67, 423 (1977). 
[10] A. Vakil and N. Engheta, Science 332, 1291 (2011).

[11] E. Forati, G. W. Hanson, A. B. Yakovlev, and A. Alù, Phys. Rev. B 89, 081410 (2014).

[12] S. A. Mikhailov, EPL 79, 27002 (2007).

[13] E. Hendry, P. Hale, J. Moger, A. Savchenko, and S. Mikhailov, Phys. Rev. Lett. 105, 097401 (2010). 